\title{
Auditing use of antibiotics in Zimbabwean neonates
}

\author{
G. Chimhini $^{a, *}$, S. Chimhuya ${ }^{a}$, L. Madzudzo ${ }^{a}$, M. Heys ${ }^{b, c}$, C. Crehan ${ }^{b}$, \\ V. Robertson ${ }^{d}$, R.A. Ferrand ${ }^{\text {e, f }}$, B. Sado ${ }^{\text {g, M. Sharland }}{ }^{\text {h }}$, A.S. Walker ${ }^{i, j}$, \\ N. Klein ${ }^{\mathrm{b}}$, F.C. Fitzgerald ${ }^{\mathrm{b}, \mathrm{f}}$
}

\author{
${ }^{a}$ Department of Paediatrics and Child Health University of Zimbabwe College of Health Sciences, Harare, Zimbabwe \\ ${ }^{\mathrm{b}}$ UCL Great Ormond Street Institute of Child Health, University College London, London, UK \\ 'Specialist Children's and Young People's Services, East London NHS Foundation Trust, London, UK \\ ${ }^{\mathrm{d}}$ Department of Medical Microbiology, University of Zimbabwe College of Health Sciences, Harare, Zimbabwe \\ e London School of Hygiene and Tropical Medicine, London, UK \\ ${ }^{f}$ Biomedical Research and Training Institute, Harare, Zimbabwe \\ ${ }^{g}$ Microbiology Department Harare Central Hospital, Harare, Zimbabwe \\ h St George's University of London, London, UK \\ 'Medical Research Council Clinical Trials Unit at UCL, University College London, London, UK \\ ${ }^{j}$ Nuffield Department of Medicine, Oxford University, UK
}

\section{A R T I C L E I N F O}

\section{Article history:}

Received 23 December 2019

Accepted 8 February 2020

Available online 19 February

2020

\section{Keywords:}

Neonatal sepsis

Low-income setting

Antibiotics

Neonatal unit

Antimicrobial stewardship

Zimbabwe

\section{S U M M A R Y}

Background: Neonatal sepsis is a major cause of morbidity and mortality in low-income settings. As signs of sepsis are non-specific and deterioration precipitous, antibiotics are often used profusely in these settings where diagnostics may not be readily available. Harare Central Hospital, Zimbabwe, delivers 12000 babies per annum admitting $\sim 4800$ to the neonatal unit. Overcrowding, understaffing and rapid staff turnover are consistent problems. Suspected sepsis is highly prevalent, and antibiotics widely used. We audited the impact of training and benchmarking intervention on rationalizing antibiotic prescription using local, World Health Organization-derived, guidelines as the standard.

Methods: An initial audit of admission diagnosis and antibiotic use was performed between $8^{\text {th }}$ May $-6^{\text {th }}$ June 2018 as per the audit cycle. An intern training programme, focusing on antimicrobial stewardship and differentiating between babies 'at risk of' versus 'with' clinically-suspected sepsis was instituted post-primary audit. Re-audit was conducted after 5 months.

Results: Sepsis was the most common admitting diagnosis by interns at both time points but reduced at repeat audit (81\% versus 59\%, $P<0.0001)$. Re-audit after 5 months demonstrated a decrease in antibiotic prescribing at admission and discharge. Babies prescribed antibiotics at admission decreased from 449 (98\%) to $96(51 \%), P<0.0001$. Inpatient days of therapy (DOT) reduced from 1243 to $1110 / 1000$ patient-days. Oral amoxicillin prescription at discharge reduced from $349 / 354(99 \%)$ to $1 \% 1 / 161(P<0.0001)$.

\footnotetext{
* Corresponding author. G Chimhini, Department of Paediatrics \& Child Health, UZCHS Parirenyatwa Hospital Complex, Mazowe Street, Harare, Zimbabwe. Tel.: +263 773553471.

E-mail address: gwenchimhini@gmail.com (G. Chimhini).
} 
Conclusion: A substantial decrease in antibiotic use was achieved by performance feedback, training and leadership, although ongoing performance review will be key to ensuring safety and sustainability.

(c) 2020 The Authors. Published by Elsevier Ltd on behalf of The Healthcare Infection Society. This is an open access article under the CC BY-NC-ND license (http://creativecommons.org/licenses/by-nc-nd/4.0/).

\section{Introduction}

The World Health Organization (WHO) estimates that infection causes $\sim 25 \%$ of 2.8 million annual neonatal deaths alongside lasting morbidity [1]. Neonatal hospital-acquired infection (HAI) is a major cause of mortality with rates in low/middle-income countries (LMICs) $\sim 3-20$ times higher than elsewhere [2]. Risks include overcrowding, poor infection control practices and reuse of single-use equipment [2]. The risk and severity of sepsis mean neonatal units are liable to overuse antibiotics. Clinicians face a difficult choice of balancing the serious risk of sepsis in this vulnerable population with the medium-long term risk of adverse effects associated with antimicrobial use, which include late-onset sepsis, necrotising enterocolitis and gut dysbiosis [3,4]. Additionally, at a population level, excessive antimicrobial use drives resistance. In Malawi, a review of blood culture isolates over nineteen years (1998-2017) showed an increase in resistance to all first-line antimicrobials from $7 \%$ to $68 \%$ in babies $<60$ days old [5]. This is especially worrying in LMICs where second- and third-line antimicrobials may be inaccessible.

Neonatal sepsis is challenging to diagnose particularly where laboratory diagnostics are lacking. Clinical features of neonatal sepsis are non-specific and clinical diagnostic algorithms such as the WHO-recommended Integrated Management of Childhood and Neonatal Illness (IMNCI) [6] algorithm maximises sensitivity over specificity. Antimicrobial stewardship programs are crucial, but often neglected, intervention for neonatal units in LMICs with limited access to second/third line antimicrobials. The optimal antimicrobial stewardship approach for neonates in LMICs is uncertain-a balance must be struck between prompt administration of antibiotics to prevent sepsis-related mortality on one hand, with minimizing individual side effects and preserving population-level therapeutic efficacy (i.e. reducing unnecessary usage) on the other. Simple tools such as audit, training and benchmarking may be useful in safely rationalising antibiotic prescription without excessive cost.

\section{Local problem}

Harare Central Hospital $(\mathrm{HCH})$, a public sector hospital in Zimbabwe, delivers 12000 babies per year. The 100-cot neonatal unit runs at $100-140 \%$ capacity and accepts nationwide surgical referrals. Overcrowding and understaffing are constant issues, as is the rapid turnover of staff, in particular, medical interns who frequently have minimal prior neonatal experience. Sepsis is a frequent admitting diagnosis, and there is a high level of concern about HAls (a recent Klebsiella outbreak had a $30 \%$ case fatality rate), in combination with a slow turnaround time for blood culture results. Although there were hard copy guidelines for first/second-line antibiotics on the ward, these were often not used. There was no formal antimicrobial stewardship programme prior to this audit.
Microbiology facilities for processing blood cultures are onsite in a different building. Blood cultures are processed manually using locally manufactured media, with limited isolate identification/susceptibility testing depending on consumable availability. Results are retrieved from the laboratory daily by intern doctors but delays are frequent. We believed antibiotic prescription rates were likely to be high and duration prolonged. In the context of severe resource constraints, any quality improvement approaches should be simple and inexpensive, utilising existing resources where possible.

We aimed to audit antibiotic use and admission diagnoses before and after performance feedback and training interventions at $\mathrm{HCH}$ neonatal unit.

\section{Methods}

A simple audit cycle was planned with feedback, education and training by senior staff as the main intervention. The standards used were local guidelines based on $W H O I M N C I$ guidelines [6] for management of suspected sepsis and included risk factors and symptoms (Supplementary Table 1). Firstline antibiotics for suspected sepsis were intravenous ampicillin or benzylpenicillin plus gentamicin. There was no indication within local guidelines for oral amoxicillin at any point.

\section{Measurement}

Baseline assessment consisted of a prospective audit of all admissions to the neonatal unit between $8^{\text {th }}$ May- $6^{\text {th }}$ June 2018 [7]. There were no specific exclusion criteria. Admitting diagnoses (as documented by the admitting intern), vital signs, examination and investigation findings and type and duration of antimicrobial therapy were recorded. Babies could have multiple diagnoses e.g. prematurity and respiratory distress. Timing of microbiological investigations carried out (e.g. pre/ post antibiotics), results and time to receipt of positive results were documented. For longer admissions, recurrent episodes of suspected sepsis and antibiotic use were documented. Participants were followed to the earliest of death, discharge, or loss to follow up. A single researcher collected anonymised data from contemporaneous clinical records using REDCap (Vanderbilt University, version 4.0.2) [8]. These data were collected as a baseline assessment prior to a large-scale quality improvement study, introducing an Android application (NeoTree [9]) as a digital quality improvement system. The antimicrobial prescription rates were so high that the interventions described below were instigated prior to NeoTree commencement.

\section{Intervention}

Audit results were fed back to unit staff and hospital executive (August 2018) after which training/education 
Table 1

Characteristics and outcome of 650 babies admitted to Harare Central Hospital at initial and repeat audit

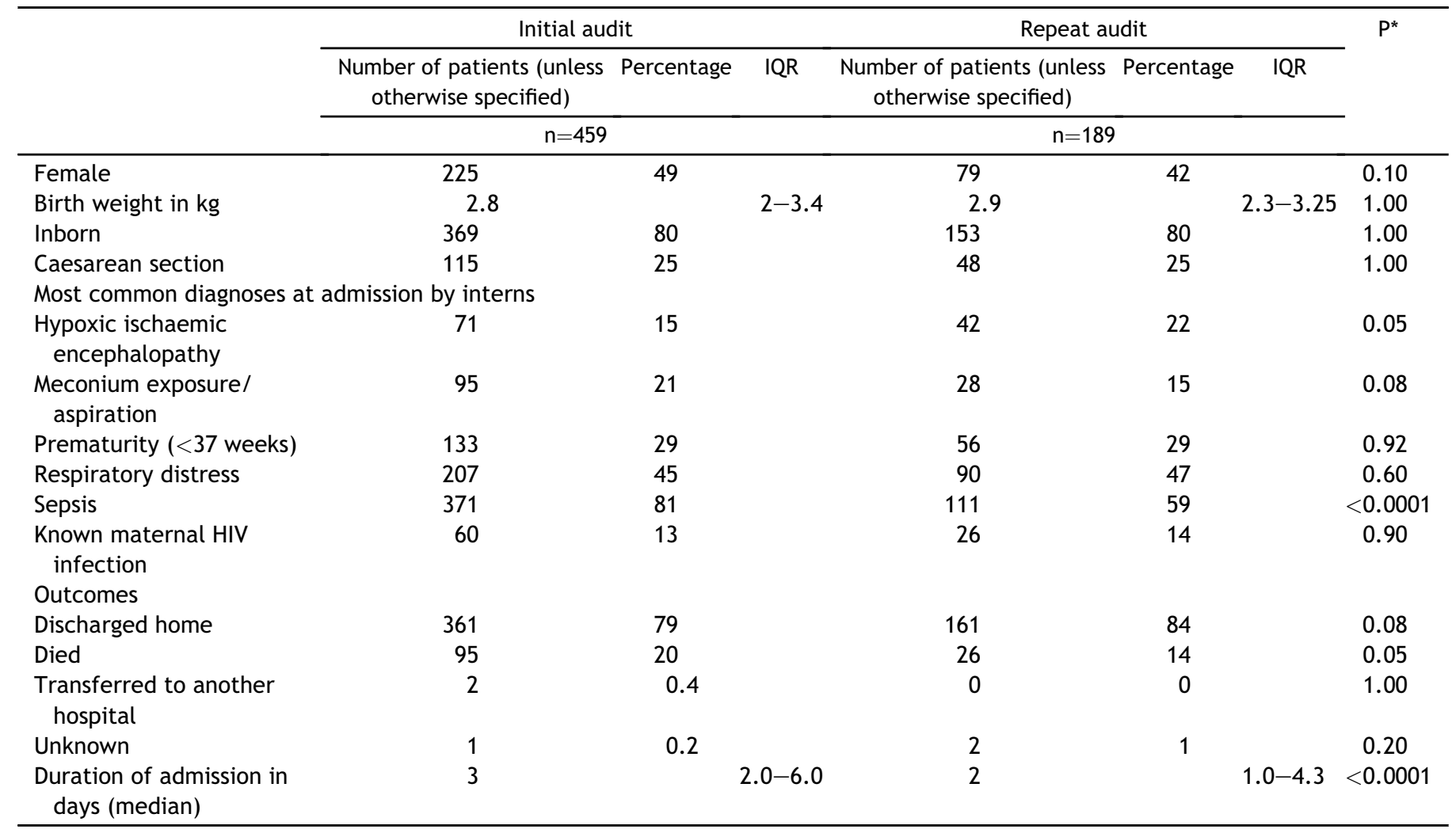

* Fisher's Exact Test, t-test or Wilcoxon rank-sum test.

interventions were instigated. Specific contextual elements considered in intervention design were the inexperience of interns in managing neonates rendering training/education key implementation tools and the potential for utilizing existing unit meetings to disseminate learning. Additionally, babies were admitted by intern doctors and reviewed by a senior paediatrician the following day, allowing rapid feedback on adherence to guidelines.

Subsequent training over three months for interns and nurses occurred. Firstly, a unit induction session by consultants for interns on antimicrobial guidelines for neonates was introduced. This was consolidated at weekly unit meetings of nursing and medical staff, disseminating key messages from the preliminary audit such as discontinuing the empiric prescription of 'prophylactic' oral amoxicillin on admission for babies not on intravenous antibiotics and at discharge. Neonatal sepsis guidelines for first-line and second-line antibiotics were displayed on the unit. Finally, antimicrobial stewardship consultant-led ward-rounds were introduced, which reinforced differentiating between babies 'at risk of' versus 'with' clinically-suspected sepsis, such that asymptomatic babies with one risk factor for sepsis were to be admitted for observation of vital signs for 24 hours before starting antibiotics. Over the course of the three months, discussions during ward meetings included clarification of criteria for starting patients on antibiotics, frequency of observation over 24 hours, discontinuation of antibiotics with negative blood culture results and antimicrobial use for cases with recurring episodes of sepsis or surgical patients transferred in from district neonatal units. Features important for the success of the intervention were clear messaging and committed leadership from the neonatal consultants and senior nursing staff, combined with the utilization of existing platforms such as the weekly unit meeting and regular teaching ward rounds. There was also nursing capacity and expertise to safely observe untreated babies with one risk factor.

\section{Intervention impact}

A repeat retrospective audit specifically reviewing antimicrobial prescriptions was conducted from $29^{\text {th }}$ October to $11^{\text {th }}$ November 2018 as above. REDCap data dictionaries for initial/repeat audit are included in Supplementary Material. A sample size of 138 would be needed at each audit to detect a decrease in prescription rates from $95 \%$ to $85 \%$ with $80 \%$ power (two-sided alpha=0.05). Other than the training as described above, no other interventions or changes to practice were instigated between audits, meaning observed outcomes were likely due to the training. Unfortunately, laboratory resource constraints meant that at repeat audit, culture results were not consistently available.

\section{Analysis}

Data were analysed in R Studio (Version 3.3.3) [10]. Characteristics between audits were compared using chi-squared tests and t-tests for categorical and continuous variables respectively. Inpatient and total days of therapy were calculated to assess antibiotic exposure [11]. The manuscript was prepared using the SQUIRE template [12]. 


\section{Ethics}

The study was approved by the Harare Central Hospital Ethics Committee (reference HCHEC 250418/48) and University College London Ethics Committee (reference 5019/004). As this was an audit assessing routine clinical care and data were anonymised, the requirement for individual consent was waived. We had no conflicts of interest.

\section{Results}

\section{Intervention delivery}

This audit cycle took place over six months from baseline assessment to repeat audit, with three months dedicated to the delivery of the training/feedback intervention. The major modification overtime was the move from the formal consultant-led induction training to less formal peer-peer training, but as regular consultant feedback during ward rounds and the weekly unit meetings continued, messaging remained consistent. A further evolution was the shift to retrospective data collection over a shorter timeframe for the repeat audit. This was partly due to earlier than the planned introduction of the NeoTree application, and partly due to staff shortages secondary to nationwide industrial action.

\section{Process measures and outcomes}

There were 648 babies included, 459 in the primary and 189 in the repeat audit (Table 1 ).

Birth weight, gestational age and the rest of demographics and clinical features were similar in the primary and repeat audit. Sepsis was the most common admitting diagnosis by interns at both timepoints but reduced at repeat audit $(81 \%$ versus $59 \%, P<0.0001$ ).

Antibiotic prescription on admission reduced significantly: 449 (98\%) versus 96 (51\%) respectively, $P<0.0001$. The most common antibiotics at admission for both timepoints were benzylpenicillin and gentamicin, prescribed to $65 \%$ and $67 \%$ respectively at primary audit and decreasing to $51 \%$ each at reaudit $(P<0.0001$ for both, Supplementary Table 2$)$. Prescription of oral amoxicillin at admission reduced from $22 \%$ to $0 \%$, the largest decrease across all antimicrobials. There was also less divergence from recommended first-line therapy: babies prescribed ceftriaxone at first admission decreasing from $9 \%$ to $1.5 \%(P=0.0004)$. Antimicrobial prescription for admissions that were not sepsis-related, such as macrosomia and safekeeping, decreased from $23 / 24(96 \%)$ to $1 / 10(10 \%)$ and $14 / 14(100 \%)$ to $0 / 4(0 \%)$ respectively.

Inpatient days of therapy (DOT) reduced from 1243 to 1110 / 1000 patient-days and the median duration of therapy reduced from 6 days (IQR 5-9) to 3 days (IQR $2-5, P<0.0001$ ) when antibiotics were given (Supplementary Table 3). Prescription of seven days of amoxicillin at discharge reduced from 349/354 (99\%) to $1 / 161(1 \%, P<0.0001)$. There was no evidence that these reductions increased mortality, which occurred in $20 \%$ versus $14 \%$ of admitted babies across the two audits respectively $(P=0.05)$. Data on antimicrobial prescription at admission were available for all babies. Outcome data including DOT were missing for three babies $(1 / 459(0.2 \%)$ at initial and $2 / 189$ (1\%) at repeat audit respectively, $P=0.20)$.
In the primary audit, blood cultures were taken for $44 \%$ $(196 / 445)$ of those starting antibiotics on admission, of which 65/196 (33\%) received results (median time-to-result 6 days, IQR 5-9) (Supplementary Results, Supplementary Table 4). Only $7 / 196(4 \%)$ cultures sent arrived in time to impact on therapy. There were 75 episodes of subsequent sepsis in 54 (12\%) admitted babies with $7 / 36(20 \%)$ positive cultures leading to treatment modification. Klebsiella pneumoniae/Lactosefermenting coliforms were common pathogens even at admission, all but one of which was resistant to third-generation cephalosporins (Supplementary Figure 1). Minimal culture data were available at repeat audit owing to resource constraints.

\section{Discussion}

In summary, sepsis was the most common admitting diagnosis on the neonatal unit and antibiotic use was high even in the absence of sepsis. Antimicrobial usage was reduced by an inexpensive training intervention.

Overall days of antibiotic therapy were higher at both time points than reported data from Chinese neonatal units (380 DOT/1000 patient-days) [11] but lower than Russia (1426 DOT/ 1000 patient-days) [13]. In general, data on DOT from African neonatal units are lacking, an evidence gap that is important to fill to allow inter-unit comparison and benchmarking to ensure that antimicrobial stewardship interventions are implemented safely and effectively. In high-income settings, reduced antibiotic usage secondary to effective stewardship has safely reduced consumption without adversely impacting mortality despite neonatal vulnerability to sepsis $[3,14]$. Timely blood culture feedback has also been shown to reduce the duration of therapy [15]. Although our unit has microbiological diagnostics onsite (unusual for LMICs), it is still vulnerable to laboratory supply and staffing shortages as experienced by other units [16]. Only $44 \%$ of babies commenced on antibiotics for suspected sepsis in the preliminary audit had a blood culture taken, and feedback of results was too slow for clinical relevance, meaning asymptomatic babies may have continued antibiotics longer than necessary.

Although our data must be interpreted cautiously due to some differences between the initial and repeat audit methodology, we observed a marked reduction in antimicrobial prescribing on our neonatal unit with simple, inexpensive methods of training, feedback and focusing on stewardship during ward-rounds. Initially, there was a prolific prescription of antimicrobials, both for babies with risk factors and/or clinical signs of sepsis, but also for diagnoses that would not usually merit antimicrobial treatment. In our unit differentiating babies with signs of sepsis who merited treatment, and those asymptomatic babies with only one risk factor who could be admitted for observation was a major feature in the training for interns and was also reinforced during wardrounds, which may have impacted on the rate of documentation of 'sepsis' at admission. Another feature of training emphasized that there was minimal evidence for 'prophylactic' or empiric antibiotics (amoxicillin) at discharge for neonates admitted to the unit.

Finally, perhaps the most effective part of the audit was the process of feedback alone. We are continuing to benchmark our progress monthly, a process that has been used to good 
effect in high- and low-income settings [11]. In particular, we are monitoring antibiotic prescription in babies who should be getting antibiotics to ensure we were not being overly conservative, withholding this crucial therapy inappropriately. Attaining a safe balance to ensure babies who needed antibiotics receive them promptly while protecting those who do not will be a challenging ongoing process.

\section{Limitations}

The limitations in this audit cycle were the prospective versus retrospective methods used in the first and second audit respectively, and the smaller sample size in the re-audit. The retrospective re-audit was used specifically to review antimicrobial use on the unit prior to a large-scale quality improvement project due to be initiated in the unit (use of NeoTree, a tablet-based digital quality improvement system with decision support [9]). It was limited to retrospective as opposed to prospective data collection due to staff shortages secondary to industrial action. Consequently, the borderline differences in mortality and admission duration between groups should be interpreted with caution. However, the differences in antimicrobial prescription rates pre/postintervention are substantial $(99 \%-1 \%, \quad P<0.0001)$, and unlikely to be explained by the difference in methods alone. Finally, we do not have follow-up data post-discharge so are unable to assess medium/long-term mortality.

In terms of generalizability, our context of having a doctorled service with frequent consultant presence on the ward to reinforce training may not be replicable in other LMIC settings. We also had nursing and spatial capacity to admit babies for observation if they had only one risk factor. However, the fact that this audit was conducted over a relatively short period, in challenging circumstances and with no additional funding for the training and education intervention, may encourage others in low-income, high-pressure settings and provide a benchmark, as well as demonstrating that simple interventions can still be effective.

\section{Conclusion}

We achieved a substantial decrease in antibiotic use with inexpensive interventions. For a low-resource hospital, the potential cost savings are also important and merit further assessment. High antibiotic use can be reduced by performance feedback, training and leadership although ongoing performance review will be key to ensuring safety and sustainability.

\section{Conflicts of interest}

We have no conflicts of interest.

\section{Funding}

Funded by the Healthcare Infection Society (reference SRG 201802 004). FF is supported by the Academy of Medical Sciences, the funders of the Starter Grant for Clinical Lecturers scheme and UCL Great Ormond Street NIHR Biomedical Research Centre. RAF is funded by the Wellcome Trust. VJR is funded by CDC-PEPFAR. ASW is supported by core support to the MRC Clinical Trials Unit [MC_UU_12023/22] through a concordat with the Department for International Development, and the Oxford NIHR Biomedical Research Centre; and is an NIHR Senior Investigator. The views expressed are those of the author(s) and not necessarily those of the NHS, the NIHR, the Department of Health or PHE.

\section{Author contribution}

Study concept and design; GC,SM,MH,CC,VR, RAF,MS,ASW, NK,FCF.

Data Collection ; GC,SC,LM,BS.FCF.

Data Analysis : VR,ASW, RAF,FCF.

Preliminary Draft: GC,ASW, RAF,NK,FCF.

Final Draft ; All Authors.

\section{Acknowledgements}

We are grateful to the staff, infants and their families at Harare Central Hospital, Dr Christopher Pasi and Matron Dade Pedzisai for their support.

\section{Appendix A. Supplementary data}

Supplementary data to this article can be found online at https://doi.org/10.1016/j.infpip.2020.100046.

\section{References}

[1] Fitchett EJ, Seale AC, Vergnano S, Sharland M, Heath PT, Saha SK, et al. Strengthening the Reporting of Observational Studies in Epidemiology for Newborn Infection (STROBE-NI): an extension of the STROBE statement for neonatal infection research. The Lancet Infectious Diseases 2016;16(10):e202-13.

[2] Zaidi AK, Huskins WC, Thaver D, Bhutta ZA, Abbas Z, Goldmann DA. Hospital-acquired neonatal infections in developing countries. The Lancet 2005;365(9465):1175-88.

[3] Makri V, Davies G, Cannell S, Willson K, Winterson L, Webb J, et al. Managing antibiotics wisely: a quality improvement programme in a tertiary neonatal unit in the UK. BMJ Open Quality 2018;7(2):e000285.

[4] Cotten CM, Taylor S, Stoll B, Goldberg RN, Hansen NI, Sánchez PJ, et al. Prolonged duration of initial empirical antibiotic treatment is associated with increased rates of necrotizing enterocolitis and death for extremely low birth weight infants. Paediatrics 2009;123(1):58-66.

[5] Iroh Tam P-Y, Musicha P, Kawaza K, Cornick J, Denis B, Freyne B, et al. Emerging resistance to empiric antimicrobial regimens for pediatric bloodstream infections in Malawi (1998-2017). Clinical Infectious Diseases 2019;69(1):61-8.

[6] World Health Organization. Integrated management of childhood and neonatal illness. Geneva: World Health Organization; 2012. Google Search [Internet]. [cited 2020 Jan 18]. Available from: https: / / www.google.com/search?client=firefox-b$\mathrm{d} \& \mathrm{q}=$ World + Health + Organization.+ Integrated + Management + of + Childhood + and + Neonatal + Illness. ++ Geneva\% $3 \mathrm{~A}+$ World + Health + Organization +2012 .

[7] Benjamin A. Audit: how to do it in practice. BMJ 2008;336(7655):1241-5.

[8] Harris PA, Taylor R, Thielke R, Payne J, Gonzalez N, Conde JG. Research electronic data capture (REDCap) - a metadata-driven methodology and workflow process for providing translational research informatics support. Journal of Biomedical Informatics 2009;42(2):377-81. 
[9] Crehan C, Kesler E, Nambiar B, Dube Q, Lufesi N, Giaccone M, et al. The NeoTree application: developing an integrated mHealth solution to improve quality of newborn care and survival in a district hospital in Malawi. BMJ Global Health 2019;4(1):e000860.

[10] Team RC. R. A language and environment for statistical computing. Vienna, Austria: R Foundation for Statistical Computing; 2017 (Version 3.4. 2)[Computer software].

[11] Lu C, Liu Q, Yuan H, Wang L. Implementation of the Smart Use of Antibiotics Program to Reduce Unnecessary Antibiotic Use in a Neonatal ICU: A Prospective Interrupted Time-Series Study in a Developing Country. Critical Care Medicine 2019;47(1):e1-7.

[12] Ogrinc G, Davies L, Goodman D, Batalden P, Davidoff F, Stevens D. SQUIRE 2.0 (Standards for Quality Improvement Reporting Excellence): revised publication guidelines from a detailed consensus process. American Journal of Critical Care 2015;24(6):466-73.

[13] Galankin TL, Kolbin AS, Sidorenko SV, Kurylev AA, Malikova EA, Lobzin YV, et al. Retrospective surveillance of antibiotic use in maternity wards and neonatal intensive care units in Saint Petersburg, Russia. European Journal of Clinical Microbiology \& Infectious Diseases 2018;37(8):1531-7.

[14] Thampi N, Shah PS, Nelson S, Agarwal A, Steinberg M, Diambomba Y, et al. Prospective audit and feedback on antibiotic use in neonatal intensive care: a retrospective cohort study. BMC Paediatrics 2019;19(1):105.

[15] Jardine MA, Kumar Y, Kausalya S, Harigopal S, Wong J, Shivaram A, et al. Reducing antibiotic use on the neonatal unit by improving communication of blood culture results: a completed audit cycle. Archives of Disease in Childhood-Fetal and Neonatal Edition 2003;88(3). F255-F255.

[16] Organization WH. reportEssential newborn care: report of a technical working group (Trieste, 25-29 April 1994). World Health Organization; 1996. those of the NHS, the NIHR, the Department of Health or PHE. 